\title{
TangibleHeart - Enabling Critical and Shared Reflections of Heart Behaviors
}

\author{
Ilhan Aslan \\ Human-Centered Multimedia Lab \\ Augsburg University \\ aslan@hcm-lab.de
}

\author{
Elisabeth André \\ Human-Centered Multimedia Lab \\ Augsburg University \\ andre@hcm-lab.de
}

\begin{abstract}
TangibleHeart is a design that seeks to encourage critical reflection by bringing to the foreground the "ugly and beautiful" ways of how one's own heart works outside explicit awareness. The artefact resembles a real heart in size and behaviour and physically beats in synchrony with the user's real heart. It allows users to experience their own heart's real-time behaviour through physical computing sensors and actuators. It can be held in ones own hands or given to the hands of onlookers for shared and contextual reflection. TangibleHeart aims to trigger a variety of feelings from users and onlookers, ranging from discomfort or uneasiness caused by the realization of the artefact's cruel embodiment to fascination and joy due to the familiar resonance produced by the artefact's behaviour.
\end{abstract}

\section{AUTHOR BIOGRAPHIES}

Ilhan Aslan is an interaction designer, engineer and educator. He has studied Computer Science at Saarland University in Germany and conducted his PhD work on movement and embodied interaction design at the Center for Human- Computer Interaction in Salzburg, Austria. He is currently employed as an "Akademischer Rat" at the University of Augsburg, Germany, where he contributes to the scholarly research and teaching at the Human-Centered Multimedia lab. He teaches lectures in Physical Computing, Human-Computer Interaction, and Usability Engineering. His research focus is in understanding the current state of human-artifact relations and to explore future alternatives.

Elisabeth André is a full professor of Computer Science and Founding Chair of Human-Centered Multimedia at Augsburg University in Germany. She has multiple degrees in Computer Science from Saarland University, including a doctorate. Elisabeth André has a long track record in multimodal human-machine interaction, embodied conversational agents, affective computing and social signal processing. She has undertaken very successful interdisciplinary collaborations with psychologists, pedagogues, medical scientists and media artists that have resulted in several awardwinning multimo-dal user interfaces, among them pedagogically wellgrounded and empirically validated learning environments for children and young people. She has been elected to several prestigious academies including her latest election to the $\mathrm{CHI}$ Academy in 2017.

\section{ABOUT THE DESIGN}

The design of TangibleHeart is inspired by Somaestehtics (e.g., Richard Shusterman (2012)) and Positive Computing (i.e., Calvo and Peters (2014)). Both (interdisciplinary) fields encourage the application of technology and design for selfreflection practices. Unlike other physiological data visualizations, TangibleHeart moves (i.e., expands) in synchrony with a user's heartbeat and thus displays the heartbeat in a more direct manner, mapping the original heart behaviour directly to a physical display. The core mechanics are computed using state of the art physical computing tools, such as an Arduino board, a pulse sensor, and a servomotor. The surface of the artefact is covert with latex.

Figure 1 presents the TangibleHeart design in two occasions, including a photo made during a workshop where TangibleHeart was showcased to foster discussion on gender and care issues.

A presenter can showcase TangibleHeart by holding a pulse sensor in one hand, for example between their thumb and index finger as it is done by the person depicted on the right hand side of Figure 1 and offer visitors to hold TangibleHeart in their hands in order to experience the presenters heartbeat. In our experience visitors not only like to experience the presenters heartbeat but also explore their own heart and compare their heart's behaviours with their friends'. Figure 2 illustrates the design process and some decisions made during the crafting process. More details of our research agenda can be found in our previous work (i.e., Aslan et. al (2016)). 

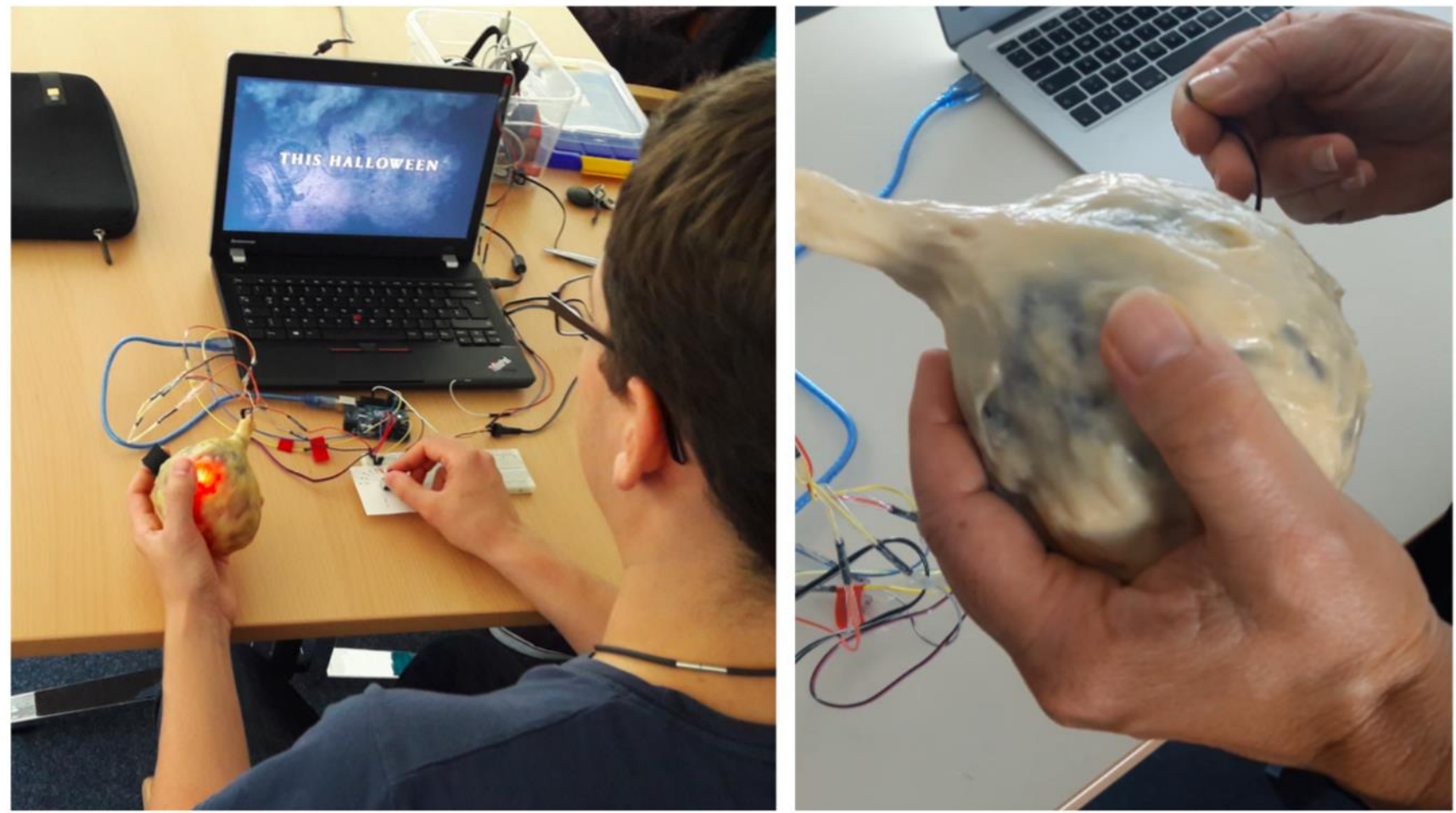

Figure 1: The TangibleHeart design is depicted in different occasions. On the left hand side TangibleHeart is being used as a tool that provides physiological feedback during watching a movie trailer. On the right hand side TangibleHeart is being showcased during a workshop, addressing gender and care issues.

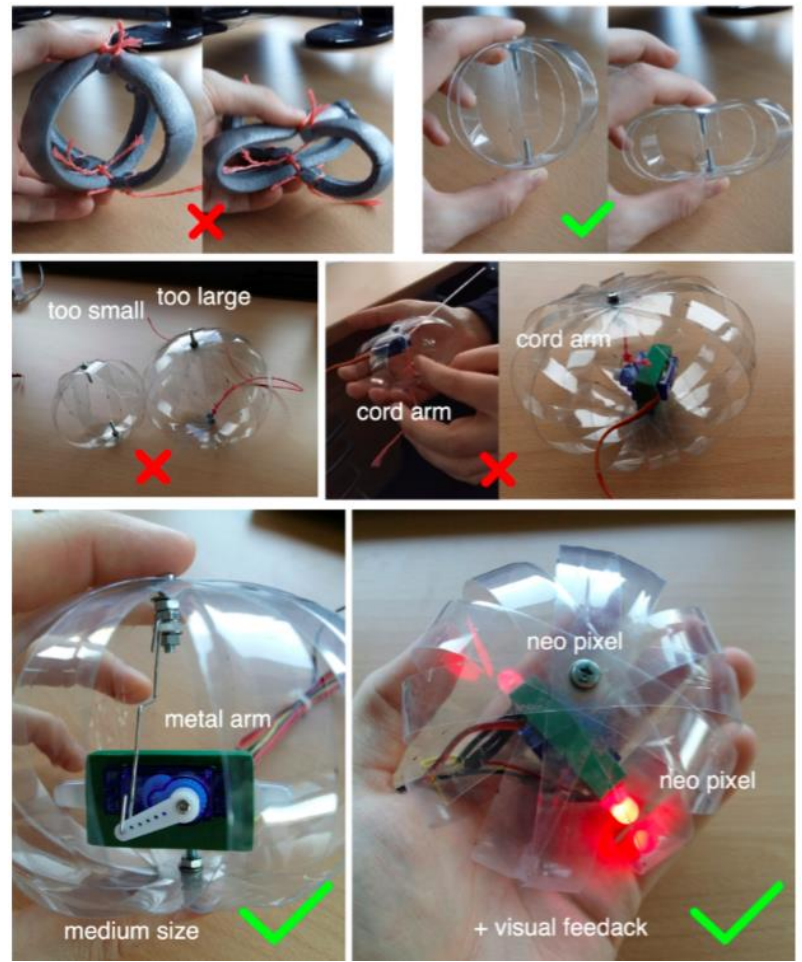

Figure 2: A glimpse at the design process, illustrating the inner workings of the prototype in earlier design phases and material explorations performed to achieve the "right" feel of a beating heart.

\section{REFERENCES}

Aslan, I. and Burkhardt, $H$. and Kraus, J. and Andre', E. (2016) Hold My Heart and Breathe with Me: Tangible Somaesthetic Designs. In Proceedings of the 9th Nordic Conference on Human-Computer Interaction (NordiCHI '16). ACM.

Rafael, A. C. and Dorian, P. (2014) Positive computing: Technology for wellbeing and human potential. MIT Press.

Shusterman, R. (2012) Thinking through the body: Essays in somaesthetics. Cambridge University Press. 